\title{
Static Envelope Patterns in Composite Resonances Generated by Level Crossing in Optical Toroidal Microcavities
}

\author{
Tal Carmon, ${ }^{1, *}$ Harald G. L. Schwefel, ${ }^{2}$ Lan Yang, ${ }^{3}$ Mark Oxborrow, ${ }^{4}$ A. Douglas Stone, ${ }^{5}$ and Kerry J. Vahala ${ }^{6}$ \\ ${ }^{1}$ Electrical Engineering and Computer Science, University of Michigan, Ann Arbor, Michigan 48109, USA \\ ${ }^{2}$, Max Planck Research Group, Institute for Optics, University of Erlangen-Nuremberg, 91058 Erlangen, Germany \\ ${ }^{3}$ Department of Electrical and Systems Engineering, Washington University, St. Louis, Missouri 63130, USA \\ ${ }^{4}$ National Physical Laboratory, Teddington, Middlesex TW11 OLW, United Kingdom \\ ${ }^{5}$ Yale University, Department of Applied Physics, New Haven, Connecticut 06520, USA \\ ${ }^{6}$ Applied Physics Department, Caltech, Pasadena, California 91125 USA
}

(Received 21 August 2007; published 13 March 2008)

\begin{abstract}
We study level crossing in the optical whispering-gallery (WG) modes by using toroidal microcavities. Experimentally, we image the stationary envelope patterns of the composite optical modes that arise when WG modes of different wavelengths coincide in frequency. Numerically, we calculate crossings of levels that correspond with the observed degenerate modes, where our method takes into account the not perfectly transverse nature of their field polarizations. In addition, we analyze anticrossing with a large avoidance gap between modes of the same azimuthal number.
\end{abstract}

DOI: 10.1103/PhysRevLett.100.103905

PACS numbers: 42.60.Da, 42.25. $-\mathrm{p}$

Zeeman and Stark realized that tuning an external field causes a variation in the position of atomic energy levels. Atomic levels can then cross each other when an applied magnetic field [1], electrical field [2], or pressure [3] is varied. Level tuning with an external field is an important tool in atomic [1] - as well as molecular-spectroscopy [4], and facilitates precise measurements such as of fine structure [1] and lifetimes [5]. Resembling behavior is known in vibrational mechanical modes [6]. In optics, anticrossings (also referred to as avoided crossings) with a gap in the transmission frequency have been reported in liquid-crystal etalons [7] and used to observe the geometric amplitude factor [8]. Crossing of optical resonances was recently reported [9] in spherical cavities.

Here we show experimentally and theoretically that optical resonances with a different number of wavelengths along the circumference can be tuned to cross in frequency. Modes of the same number of wavelengths, on the other hand, are shown to anticross with a large gap. Overall, diverse azimuthal and radial mode shapes are imaged, and modes with complex transverse shapes and polarizations are calculated. Using a fluorescent mode-mapping technique enabled imaging of noncircular mode patterns that signals crossing. This visual indication was hidden when we were using bare nonfluorescent cavities.

Near the degeneracy region, modes exhibiting a different number of circumferential wavelengths are simultaneously excited with a single-frequency laser source to produce a standing interference envelope. Here, only modes circulating in one direction are examined. Level crossing of countercirculating modes is possible [10] but, in distinction to the relatively large spatial "beats" observed here, manifests itself as a fine standing-wave interference pattern with nodes lying just half an optical wavelength apart. Moreover, in experiments, countercirculating modes (of similar indices) are typically split (i.e., nondegenerate) [11-13] due to imperfect isotropy (broken axial symmetry). In our system, this split $\left(\sim 10^{7} \mathrm{~Hz}\right)$ is much smaller than the free spectral range $\left(\sim 10^{12} \mathrm{~Hz}\right)$; such a splitting and the type of anisotropy it originates from might therefore play only a minor role here in bringing levels close enough to cross.

Optical resonators in general [14-16] and dielectric whispering-gallery (WG) cavities [17,18] in particular have a set of discrete optical eigenfrequencies. For a geometry in which the wave equation is separable, such as a cylindrical or spherical resonator, each mode can be labeled by three integer indices. Normally, by convention, the resonance frequency increases monotonically with each mode index. In a dielectric ring, for example, a whispering-gallery mode with a fixed and small transverse mode index and a large azimuthal mode index of $m=105$, say, will have 105 wavelengths along the circumference and will resonate at an optical frequency higher than that of

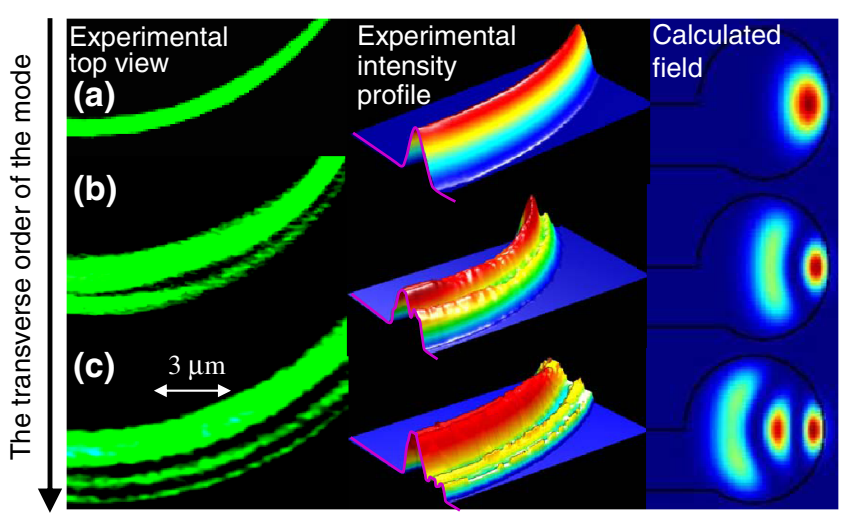

FIG. 1 (color online). Experimental results: Top view of modes with increasing transverse index. On the right section of the table, the calculated field profile in a cross section perpendicular to the mode's azimuthal direction of propagation. 
a mode with 100 wavelengths along the circumference. Hence, in general, the simultaneous excitation of both of these modes will require two separate laser sources, and their resulting interference envelope will circulate around the ring at typically millions of revolutions per second. Such rapidly moving interference patterns are not investigated here. If we vary the discrete transverse mode index in such a system, two levels with different $m$ indices could lie close in frequency, but such coincidences, i.e., degeneracies, would be accidental. In the toroidal resonators, in contrast, one has a continuous (dimensionless) parameter, namely, the aspect ratio of the major to minor diameters of the torus [19], which can be varied leading to degeneracy and potentially strong mode mixing.

The wave equations for the toroidal resonator [20] are partially separable. In the azimuthal direction, the rotation symmetry allows an $\exp (\operatorname{im} \phi)$ dependency to be factored out. Modes of different $m$ are hence the solutions of two independent equations and can cross each other. The eigenfunctions in this transverse plane cutting through the torus (see Fig. 2 below) are labeled here by only a single mode index, which we shall refer to as the "transverse" index. When the aspect ratio is varied, modes of the same $m$ and different transverse index interact and can generate avoided crossings.

In this work, level crossing refers to a situation when the modes lie close enough in frequency to be simultaneously excited by the same single-frequency laser source [21]. We find both experimentally and theoretically that the frequency difference between modes with different azimuthal indices $m$ can be completely compensated by changing the transverse index. Modes of different transverse indices extend differently into the air clad and hence their resonance frequencies have different slopes with respect to changes in the aspect ratio. Having many levels with different slopes implies crossings. Because of their difference in azimuthal index, the superposition of two such degenerate modes at a crossing exhibits an azimuthally modulated envelope, i.e., one that beats circumferentially. As modes are resonating at the same frequency, the azimuthally modulated envelope is stationary.

The experimental setup consists of a high- $Q$ toroidal cavity [22], fabricated from silica on a silicon wafer. Optical coupling of the continuous-in-time pump laser into the cavity is performed via a tapered fiber [23-25]. In order to make the IR composite-mode pattern visible, the cavity is doped with erbium [26,27], which converts photons stored in the $1500 \mathrm{~nm}$ mode into $500 \mathrm{~nm}$ (green) light via 3-photon fluorescent up-conversion. Advantages of up-conversion mode mapping [26] include improvement of the optical resolution by a factor of 3 and a uniform detection sensitivity in the cavity volume. Additionally, although the examined optical mode is at the telecom compatible (vacuum) wavelength of $1500 \mathrm{~nm}$, the indication for its intensity (when using the 3-photon upconversion) is in the visible where high-quality microscopes and cameras are available. Other known modemapping techniques include the near-field probe [28] method. To interpret the experimental images, we also calculate numerical solutions for the resonances of the torus, shown in Figs. 1-3.

Fundamental and high-order transverse modes (not crossing at this stage) are photographed in Fig. 1 where they are presented side by side with their calculated field
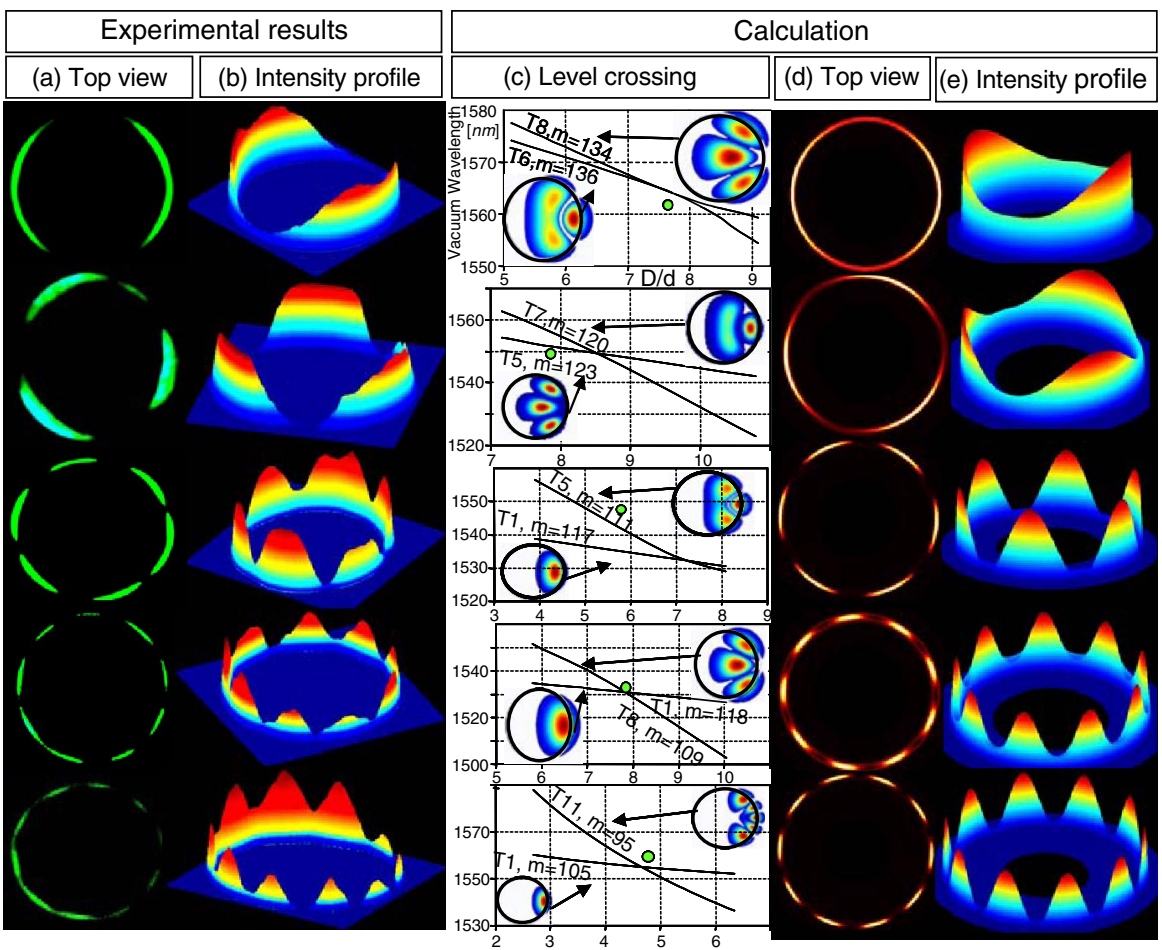

FIG. 2 (color online). Crossed modes. (a) Top-view image of the measured mode. (b) Intensity profile of the measured mode. (c) Graph of the calculated resonance frequency (given in vacuum wavelength units) versus the ratio of the major and minor diameter for two different modes with similar polarization. $T x$ stands for the $x$ transverse mode. $m$ describes the number of optical waves resonating along circumference [the field changes as to where the azimuthal coordinates]. The green circle represents the experimentally measured vacuum wavelength and shape. (d),(e) are like (a),(b) but based on calculation. 
for comparison. It is interesting to note that the ring-to-ring distance in Fig. 1(c) is subwavelength. Thanks to the upconversion mapping technique it can, however, still be imaged. Figure 1 demonstrates that high-order transverse modes do exist even in a relatively thin toroid.

We now discuss the composite modes that consist of different fundamental and high-order modes (such as the ones shown in Fig. 1). Figure 2 shows several such composite modes, where the number of maxima recorded (thus wavelength difference along the circumference) changes between 2 and 10 depending on the cavity geometry and the input optical frequency. Correspondingly, the wavelength difference along the circumference changes from 2 to 10 . The results in Fig. 2 are in agreement with numerically calculated crossings. Deviation between the calculated crossing (line intersection in Fig. 2(c)] and the experimental loci of crossing [circle in Fig. 2(c)] lies within the experimental uncertainties.

The composite modes at crossings do not only have maxima and nodes in the azimuthal direction; in Fig. 3, we show modes with both radial and azimuthal maxima in the interference envelope. We imaged up to three maxima in the radial direction and 18 maxima in the azimuthal direction [Fig. 3(b)]. In general, a higher difference of the azimuthal index, $\Delta m$, requires a higher radial index difference in order to compensate the frequency difference and lead to a composite mode; an azimuthal index difference of seven is compensated by a radial index difference of two [Fig. 3(a)], and an azimuthal difference of 18 needs a radial index difference of three to compensate [Fig. 3(b)]. Note also that the maxima in Fig. 3 are arranged in a "zigzag" pattern, which reflects the tendency of the rings in the constituent radial mode to be $\pi$ out of phase (for such rings, see Fig. 1).
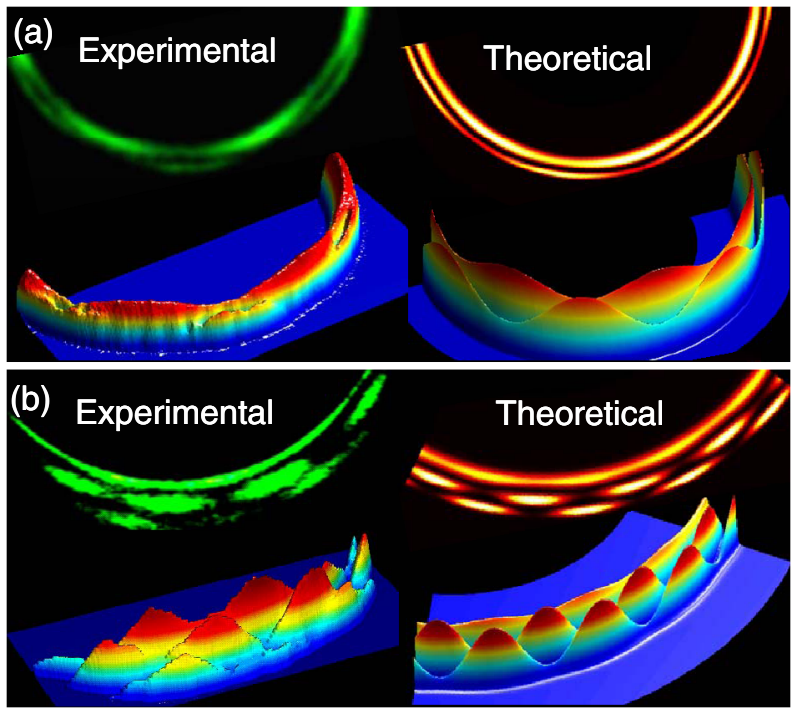

FIG. 3 (color online). Crossed modes with azimuthal as well as radial profiles. (a) Composed of fundamental modes similar to the first two shown in Fig. 1. (b) Composed of modes similar to the first and the last modes shown in Fig. 1.
In a torus exhibiting perfect axial symmetry, composite modes like those in Fig. 2 have no preferred azimuthal orientation. For any given composite mode, there thus exists a family of others with the same frequency but with an envelope pattern that is rotated arbitrarily. In an experimental system, however, the relative phase of the two degenerate traveling whispering-gallery modes is "pinned" by a nonperfect axial symmetry. Causes are likely to be deviations of the cavity geometry from a perfect ring as well as azimuthal variation in the refractive index. To give a scale, deviations in the shape of the cavity from that of a perfect ring are about $1 \%$ of the radius. Coupling the light into the cavity can also cause a breaking of the axial symmetry. Yet, in a high- $Q$ cavity only a relatively small transmission at the coupler region is necessary to achieve critical coupling (to give a scale, the coupler here is $\sim 1$ micron away from the cavity and hence creates only a weak anisotropy). Therefore, as expected, flipping the coupler position to the other side of the toroid as well as varying the toroid-to-coupler distance did not appear to affect the orientation of the observed modes.

Another family of resonances is the set of transverse modes within each of the separable families, each member of this set has the same azimuthal index. For such modes with identical $m$ [Fig. 4(a)], the remaining cylindrical coordinates $(\rho, z)$ are in general not separable. Coupling between the governing equations results [29] in anticrossing that is characterized by a gap between resonances [Fig. 4(c)] and mode evolution via state of a mixed shape and polarization at the avoidance region [Fig. 4(b) and 4(d)]. In more detail, the curves appear as if they should cross, but then repel each other on approach, to veer away, with each taking the path, the shape, and the polarization of the other. It is interesting to note that the size of the gap [Fig. 4(a)] varies from one crossing to another. We use here a "2.5-D" finite-element method [29] that does not constrict the WG mode's polarization to be either purely transverse magnetic (TM) or purely transverse electric (TE). Looking towards nonlinear and QED effects, our method can thus accurately simulate the quasi-TM and quasi-TE modes of even the smallest spheres or toroids with low azimuthal mode orders and tiny mode volumes.

In conclusion, since the frequency difference between component WG modes as in Fig. 2 is zero $(\Delta \omega=0)$ while their wave-number difference $\left[\Delta k=2 \pi\left(1 / \lambda_{1}-1 / \lambda_{2}\right)\right]$ is finite, the velocity, $V=\Delta \omega / \Delta k$, of the envelope necessarily vanishes, creating an interference pattern that is frozen in space. Practically, to coexcite such modes with a single pump, the modes should lie closer in frequency than their bandwidth. Continuous improvements in optical cavities suggests that level crossing will soon be reported in other types of devices such as disks [30]. The coupling of near-lying optical modes within a single device by scattering [13], gratings [31], or other techniques, could facilitate applications such as filters [32], gyroscopes [33], and delays [34]. Until now, it was largely thought that these applications will require multicavity configurations [35- 


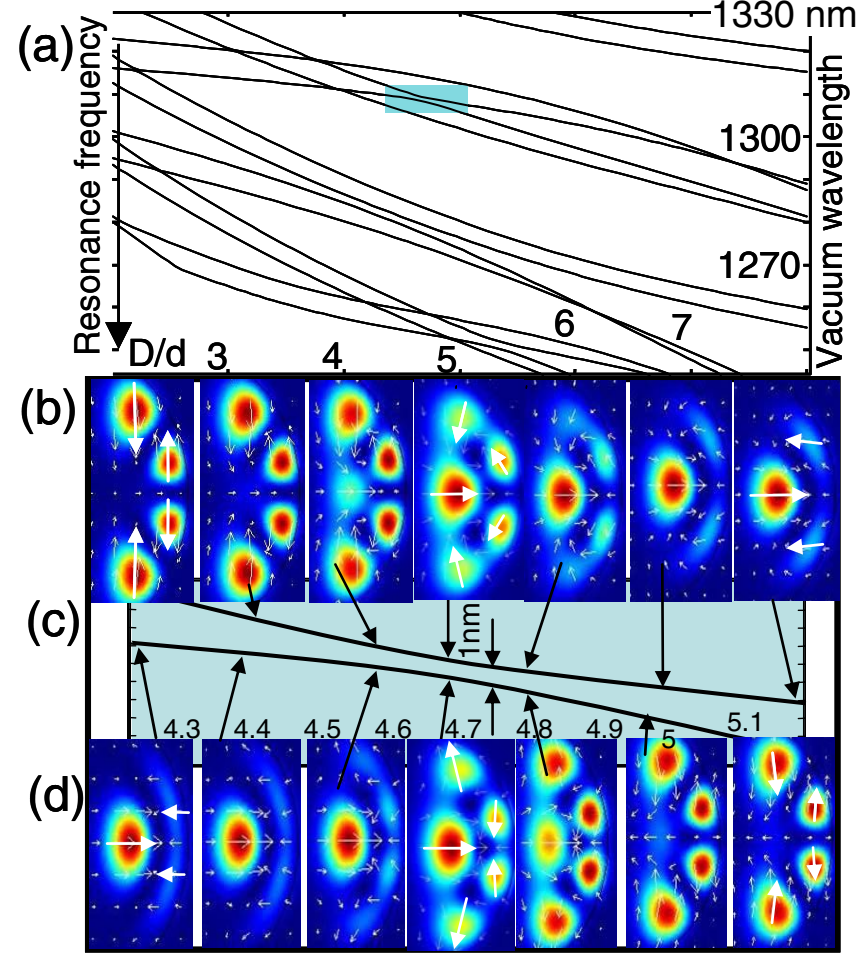

FIG. 4 (color online). Calculated anticrossing for modes of similar azimuthal index. (a) Resonance frequency as a function of the ratio between major and minor diameter $(m=106$, external diameter $=17.5 \mu \mathrm{m}$, refractive index 1.46). (b),(d) Evolution of the transverse mode structure at the marked region in (a), arrows indicate the magnetic field direction. (c) Zoom-in to the marked region in (a).

37]. The work described here, however, suggests that, by exploiting level crossings, they could each be realized within a single microcavity. Going from many resonators to many nearby resonances on the same device is trading off the nontrivial coupling between modes with the simplicity of dealing with a single photonic device.

We acknowledge discussion with A. A. Savchenkov, I. Bucher, J. Scheuer, I. Klich, O. Painter, and M. Eichenfield; and support from Caltech Lee Center and DARPA. H.G.L.S. acknowledges financial support of L. J. Wang, A. D.S. acknowledges support of NSF Grant No. DMR-0408636. M. O. acknowledges support from the NMS Pathfinder Metrology Programme.

*tcarmon@umich.edu; http://www.eecs.umich.edu/ tcarmon/

[1] F. D. Colegrove et al., Phys. Rev. Lett. 3, 420 (1959).

[2] W. F. Polik, D. R. Guyer, and C. B. Moore, J. Chem. Phys. 92, 3453 (1990).

[3] P. T. C. Freire, O. Pilla, and V. Lemos, Phys. Rev. B 49, 9232 (1994).
[4] R. N. Zare, J. Chem. Phys. 45, 4510 (1966).

[5] Rw. Schmiede et al., Phys. Rev. A 2, 1216 (1970).

[6] J.R. Kuttler and V. G. Sigillito, J. Sound Vib. 75, 585 (1981).

[7] J. S. Patel and Y. Silberberg, Opt. Lett. 16, 1049 (1991).

[8] D. Bouwmeester et al., J. Mod. Opt. 43, 2087 (1996).

[9] A. A. Savchenkov et al., Phys. Rev. A 76, 023816 (2007).

[10] One way of doing so is letting light to propagate in both directions of the tapered coupler instead of one direction as in here.

[11] J. C. Swindal et al., Opt. Lett. 18, 191 (1993).

[12] M.L. Gorodetsky, A. D. Pryamikov, and V.S. Ilchenko, J. Opt. Soc. Am. B 17, 1051 (2000).

[13] T. J. Kippenberg, S. M. Spillane, and K. J. Vahala, Opt. Lett. 27, 1669 (2002).

[14] Y. Yamamoto and R. E. Slusher, Phys. Today 46, No. 6, 66 (1993).

[15] R. K. Chang and A.J. Campillo, Optical Processes in Microcavities (World Scientific, Singapore; New Jersey, 1996), p. x.

[16] K. J. Vahala, Nature (London) 424, 839 (2003).

[17] A. B. Matsko and V.S. Ilchenko, IEEE J. Sel. Top. Quantum Electron. 12, 3 (2006).

[18] V.S. Ilchenko and A. B. Matsko, IEEE J. Sel. Top. Quantum Electron. 12, 15 (2006).

[19] Experimentally, many cavities are made where the ratio changes discreetly.

[20] F. Cap and R. Deutsch, IEEE Trans. Microwave Theory Tech. 26, 478 (1978).

[21] Typical widths in this work are $300 \mathrm{kHz}$ for the laser source and lines narrower than $200 \mathrm{MHz}$ for the resonances.

[22] D. K. Armani et al., Nature (London) 421, 925 (2003).

[23] J. C. Knight et al., Opt. Lett. 22, 1129 (1997).

[24] M. Cai, O. Painter, and K. J. Vahala, Phys. Rev. Lett. 85, 74 (2000).

[25] T. Carmon et al., Opt. Express 15, 7677 (2007).

[26] L. Yang and K. J. Vahala, Opt. Lett. 28, 592 (2003).

[27] L. Yang, D. K. Armani and K. J. Vahala, Appl. Phys. Lett. 83, 825 (2003).

[28] J. C. Knight et al., Opt. Lett. 20, 1515 (1995).

[29] M. Oxborrow, IEEE Trans. Microwave Theory Tech. 55, 1209 (2007).

[30] B. Gayral et al., Appl. Phys. Lett. 75, 1908 (1999).

[31] V.S. Ilchenko et al., in Conference on Lasers and Electro Optics (Optical Society of America, Baltimore, 1999), p. 67.

[32] C.K. Madsen and J.H. Zhao, Optical Filter Design and Analysis: A Signal Processing Approach (Wiley, New York, 1999), p. xiii.

[33] J. Scheuer and A. Yariv, Phys. Rev. Lett. 96, 053901 (2006).

[34] J. K. S. Poon et al., J. Opt. Soc. Am. B 21, 1665 (2004).

[35] A. Yariv et al., Opt. Lett. 24, 711 (1999).

[36] N. Stefanou and A. Modinos, Phys. Rev. B 57, 12127 (1998).

[37] A. Melloni, F. Morichetti, and M. Martinelli, Opt. Quantum Electron. 35, 365 (2003). 